\title{
Comparative Experimental and Numerical Investigation on Electrical Discharge Drilling of AISI 304 using Circular and Elliptical Electrodes
}

\author{
Ali Tolga Bozdana1,* - Nazar Kais Al-Kharkhi² \\ ${ }^{1}$ University of Gaziantep, Mechanical Engineering Department, Turkey \\ 2 University of Baghdad, Automated Manufacturing Engineering, Iraq
}

This work introduces a new electrode geometry for making holes with high aspect ratios on AISI 304 using an electrical discharge drilling (EDD) process. In addition to commercially available cylindrical hollow electrodes, an elliptical electrode geometry has been designed, manufactured, and implemented. The principal aim was to improve the removal of debris formed during the erosion process that adversely affects the aspect ratio, dimensional accuracy, and surface integrity. The results were compared and discussed to evaluate the effectiveness of electrode geometry on the machining performance of EDD process with respect to the material removal rate (MRR,) the electrode wear rate (EWR), and the tool wear ratio (TWR). Dimensional features and surface characteristics of the drilled holes were also evaluated in aspects of overcut (OC), aspect ratio (AR), and surface roughness (SR). The experimental results revealed that an elliptical electrode is preferable for producing holes with good dimensional accuracy and improved surface quality. In addition, 3D models of flow field with liquid, gas, and solid phases are proposed for analyses of the interelectrode gap within the machining zone. Based on the results obtained from models, the elliptical electrode also exhibited good flushing capability for effective debris removal.

Keywords: electrical discharge drilling, stainless steel, electrode geometry, drilling performance, dimensional accuracy, surface quality

Highlights

- A new electrode with elliptical geometry was implemented for the drilling of stainless steel using an EDD process.

- A number of drilling operations were conducted using circular and elliptical electrodes to produce $\emptyset 3 \mathrm{~mm}$ holes.

- The effectiveness of both electrode geometries on drilling performance and hole characteristics was compared.

- $3 D$ three-phase CFD models were developed to analyse flushing and debris removal at interelectrode gaps.

\section{INTRODUCTION}

Stainless steel has been widely used in many medical fields, defence, chemical, construction, and aerospace due to its characteristics of good corrosion resistance, low thermal conductivity, and high strength at elevated temperatures [1]. Among other grades of stainless steels, AISI 304 SS is difficult to drill with conventional methods, and the electrical discharge drilling (EDD) process has recently been used for this purpose. EDD is an electro-thermal process used for drilling small holes on electrically conductive materials, based on the eroding effect of electric sparks occurring between the tool electrode and workpiece [2]. Drilling operations are conducted using tubular (hollow) electrodes through which dielectric fluid is flowing for washing removed particles (debris) away from the machining zone. Adequate flushing at interelectrode gap is significant in drilling holes with high aspect ratios. In such case, the proper circulation of dielectric and effective debris removal are difficult to achieve, which affect drilling performance and hole features.

Numerous methods have been devised to improve flushing in EDD applications. Some researchers have tried to manipulate the relative movement between electrode and workpiece to stimulate debris removal. The orbital movement of the tool electrode in a radial direction was employed for manipulating the side interelectrode gap [3] and [4]. The bottom interelectrode gap was also manipulated by applying ultrasonic vibrations in the vertical direction [3], [5] and [6]. Moreover, it is reported that increasing electrode rotation [7] to [9] and dielectric pressure [10] to [12] have provided improvements in flushing and drilling performance. In contrast, only a few studies have been conducted for investigating the effect of electrode shape in the EDD process. Nastasi and Koshy [13] added geometric features of helical and radial slots on cylindrical copper electrodes to improve the gap flushing in drilling of 6061 aluminium alloy. Plaza et al. [14] studied the effects of helix angle and flute depth on helical electrodes to improve debris removal while increasing the hole depth in the drilling of Ti-6Al-4V. Another study on using helical electrodes with the implementation of ultrasonic vibrations on workpiece was conducted by Hung et al. [15] in the drilling of Hymu 80 nickel alloy. 
In addition to experimental works, there are few studies on modelling the flow characteristics at electrode-workpiece interaction. Nastasi and Koshy [13] modelled the flow fields in frontal and lateral gaps using ANSYS CFX to optimize the tool electrode comprising geometric features. Xie et al. [16] presented a 2D flow model developed using CFD in an ultrasonically assisted EDM process. Their model involved phases of kerosene dielectric and debris at the bottom and side regions of the interelectrode gap. Wang and Han [17] and [18] proposed a 3D model of flow field with liquid, gas, and solid phases to analyse the machining gap during electrode jump in EDM. The variations in bubble volume at the interelectrode gap in ultrasonically assisted EDM were studied by Kong et al. [19]. The 3D geometrical modelling of the flow field at interelectrode gap was established using FLUENT software. In another study of Xie et al. [20], a 2D model of the flow field in ultrasonic-assisted EDM was constructed with liquid and debris phases. Debris distribution and velocity variations at the bottom and side gaps during the ultrasonic vibration cycle were investigated. Zhang et al. [21] developed a 2D model to simulate the movement and distribution of debris in EDM with self-adaptive electrode movement. The results indicated that the quantity of debris flushed away was limited, and most of the debris in the gap was aggregated at the interelectrode gap. It should be noted that there is no comprehensive work in related literature on the modelling of flushing and debris removal in the EDD process.

This study presents experimental and numerical investigations of the drilling of stainless steel 304 using circular and elliptical electrodes. Several drilling operations were performed to examine the effectiveness of electrode geometry on machining performance as well as the dimensional accuracy and surface quality of drilled holes. Numerical analyses were also carried out based on 3D three-phase CFD models for the simulation of flushing capabilities of electrodes. The results were compared and discussed in detail.

\section{METHODS AND MEASUREMENTS}

Tubular brass electrodes with two different geometries (Fig. 1) were used for drilling holes. The circular electrode is commercially available, and the elliptical electrode was manufactured with the forging of the circular electrode. It was designed so that both electrodes have identical inner and outer diameters of $\varnothing 1 \mathrm{~mm}$ and $\varnothing 3 \mathrm{~mm}$, respectively. It should be noted that the elliptical electrode was provided additional gaps of $0.1 \mathrm{~mm}$ on each side. Thus, the elliptical electrode has a diameter of $2.8 \mathrm{~mm}$ on one side and 3 $\mathrm{mm}$ on the other side, resulting in the drilling of holes with the same diameter as the circular electrode.
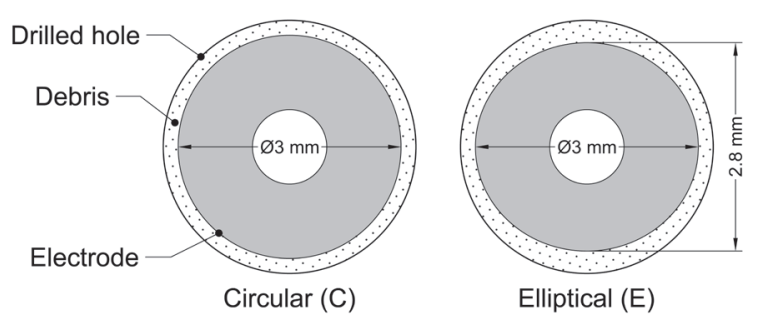

Fig. 1. Electrode geometries

Drilling operations were conducted on a JS AD20 EDD machine (Fig. 2). Vertical movement of the tool electrode was achieved with servo-control, while axis movements were shown on the coordinate display. Machining settings were selected on the control panel. Dielectric fluid was filtered and pumped through the hollow electrode throughout the drilling process.

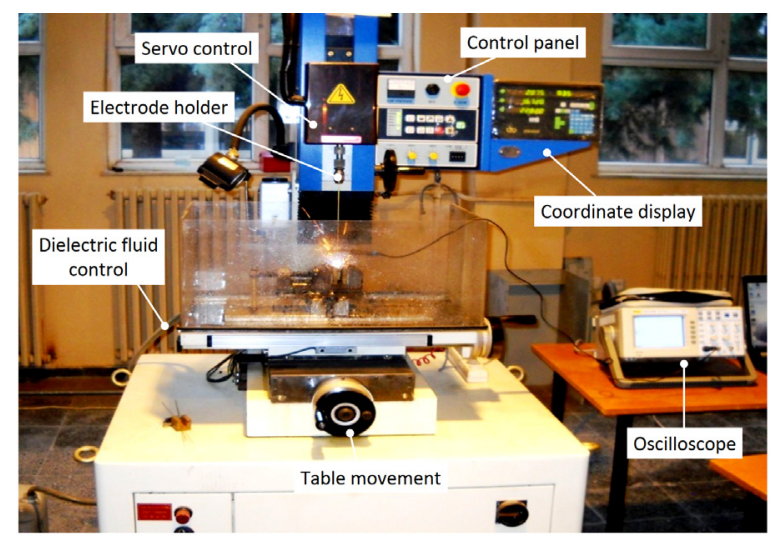

Fig. 2. EDD machine with experimental setup

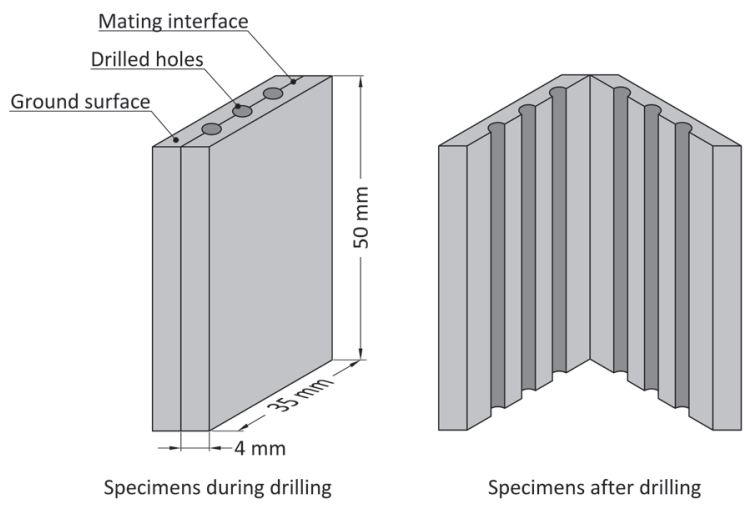

Fig. 3. Sketch of specimens

Holes were drilled on specimens of AISI 304 (Fig. 3). They were produced on the mating interface 
of the specimens, and then separated after drilling to perform measurements on the hole surfaces.

Table 1 presents the machining conditions. They were selected based on preliminary experiments. Five holes using both electrodes were drilled in durations of $(1,2,3,4$, and 5$) \mathrm{min}$.

Table 1. Drilling parameters

\begin{tabular}{lc}
\hline Peak current: & $24 \mathrm{~A}$ \\
\hline Pulse-on time: & $44 \mu \mathrm{s}$ \\
\hline Pulse-off time: & $5 \mu \mathrm{s}$ \\
\hline Voltage: & $50 \mathrm{~V}$ \\
\hline Capacitance: & $1476 \mu \mathrm{F}$ \\
\hline Dielectric fluid: & Deionized water \\
\hline Dielectric pumping pressure: & $50 \mathrm{~kg} / \mathrm{cm}^{2}$ \\
\hline Electrode rotation speed: & $200 \mathrm{rpm}$ \\
\hline
\end{tabular}

Process performance was evaluated using the material removal rate $(M R R)$ and electrode wear rate $(E W R)$, as given in Eqs. (1) and (2), respectively. Initial and final weights of specimens $\left(W_{s(i)}\right.$ and $\left.W_{s(f)}\right)$ and electrode $\left(W_{e(i)}\right.$ and $\left.W_{e(f)}\right)$ were weighed using a digital scale with a precision of $\pm 1 \mathrm{mg}$. The drilling time $(t)$ was recorded with a precision timer. The tool wear ratio (TWR) was also employed to evaluate the dimensionless relative effect between electrode wear and material removal (Eq. (3)).

$$
\begin{gathered}
\operatorname{MRR}\left[\frac{\mathrm{g}}{\min }\right]=\frac{\left(W_{s(i)}\right)-\left(W_{s(f)}\right)}{t}, \\
E W R\left[\frac{\mathrm{g}}{\min }\right]=\frac{\left(W_{e(i)}\right)-\left(W_{e(f)}\right)}{t}, \\
T W R=\frac{E W R}{M R R} .
\end{gathered}
$$

The dimensions of the drilled holes were determined based on high-resolution photographs. Because of side-sparking between the electrode and workpiece, the diameter of the drilled hole $\left(D_{h}\right)$ is always larger than the electrode diameter $\left(D_{e}\right)$. This is called Overcut $(O C)$, which was calculated based on enlargement in the whole diameter with respect to the electrode diameter (Eq. (4)). The aspect ratio $(A R)$ of the drilled holes was also considered as a measure of the erosion capability of electrodes, as given in Eq. (5) where $\left(H_{h}\right)$ refers to the depth of the drilled holes.

$$
\begin{gathered}
O C[\%]=\frac{D_{h}-D_{e}}{D_{e}} \times 100, \\
A R=\frac{H_{h}}{D_{h}} .
\end{gathered}
$$

The surface quality of holes was evaluated based on surface roughness $(S R)$. The roughness average $\left(R_{a}\right)$ was measured on each hole surface using a Mitutoyo SJ-401 roughness tester with a cut-off length of $0.8 \mathrm{~mm}$. Measurements were repeated three times at different regions on each hole surface, and the average of readings was taken as the roughness value.

In addition, the surface topography of the drilled holes was analysed using micrographs taken with Inspect S50 SEM. Micrographs were taken at the identical magnification and energy levels of $645 \mathrm{X}$ and $30 \mathrm{kV}$, respectively.

\section{EXPERIMENTAL RESULTS AND DISCUSSIONS}

Fig. 4 shows the photographs of drilled holes using circular and elliptical electrodes at drilling times of $(1,2,3,4$, and 5) min. Table 2 presents the results of $M R R, E W R, T W R, O C, A R$, and $S R$ after drilling

\begin{tabular}{|c|c|c|c|c|c|c|c|c|c|c|c|c|c|c|c|c|}
\hline \multirow{2}{*}{$\begin{array}{l}\text { Hole } \\
\text { No. }\end{array}$} & \multicolumn{2}{|c|}{$D_{h}[\mathrm{~mm}]$} & \multicolumn{2}{|c|}{$H_{h}[\mathrm{~mm}]$} & \multicolumn{2}{|c|}{ MRR [g/min] } & \multicolumn{2}{|c|}{ EWR [g/min] } & \multicolumn{2}{|c|}{$T W R$} & \multicolumn{2}{|c|}{ OC [\%] } & \multicolumn{2}{|c|}{$A R$} & \multicolumn{2}{|c|}{$S R[\mu \mathrm{m}]$} \\
\hline & $C$ & $E$ & $C$ & $E$ & $C$ & $E$ & $\mathrm{C}$ & $E$ & $C$ & $E$ & $C$ & $E$ & $C$ & $E$ & $C$ & $E$ \\
\hline 1 & 3.45 & 3.17 & 10.06 & 12.86 & .770 & 0.710 & 0.093 & 0.160 & 0.121 & 0.225 & 15.00 & 5.67 & 2.92 & 4.06 & 4.25 & 3.81 \\
\hline 2 & 43 & .16 & 7.02 & 22.31 & 0.611 & 0.615 & 0.100 & 0.150 & 0.164 & 0.243 & 14.33 & 5.33 & 4.96 & 7.10 & 4.24 & 3.78 \\
\hline 3 & 45 & & & & .538 & & .110 & & 0.204 & 0.246 & 15.00 & 5.6 & 7.00 & 9.27 & 4.04 & 3.67 \\
\hline 4 & 42 & & 3.04 & 35.03 & 0.498 & 0.497 & 0.113 & 0.125 & 0.226 & 0.252 & 14.00 & 4.67 & 9.70 & 11.16 & 3.87 & 3.55 \\
\hline 5 & 3.41 & 3.19 & 37.49 & 42.06 & 0.470 & 0.490 & 0.112 & 0.124 & 0.238 & 0.253 & 13.67 & 6.33 & 11.00 & 13.20 & 3.89 & 3.58 \\
\hline
\end{tabular}
operations.

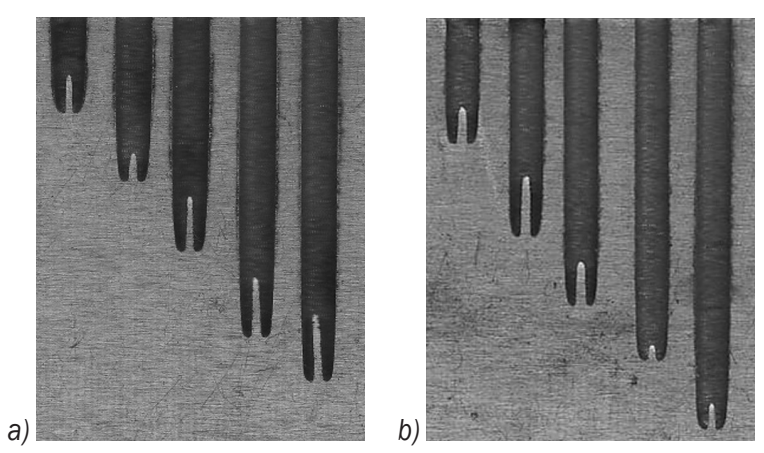

Fig. 4. Photographs of drilled holes; a) circular, b) eliptical

Table 2. Experimental results obtained with circular (C) and elliptical (E) electrodes 


\subsection{Drilling Performance}

$M R R$ is a significant output in the EDD process due to its vital influence on the engineering economy. Higher $M R R$ values indicate greater amounts of material removal per unit time. The results of $M R R$ for circular and elliptical electrodes are compared in Fig. 5. The elliptical electrode provides higher $M R R$ in comparison to the circular electrode, resulting in deeper holes. This is because the spark intensity was increased, and flushing was improved in the case of the elliptical electrode. In other words, the elliptical electrode promotes extra space for debris removal and flushing supply. Thus, greater amount of debris will flow away from the interelectrode gap, and higher $M R R$ is obtained. It is also observed that $M R R$ values for the elliptical electrode are not diminished by certain hole depths whereas material removal with a circular electrode becomes lesser by increasing the drilling depth. Moreover, the $M R R$ curves in Fig. 5 for both electrodes decrease with drilling depth since flushing the debris away from the machining zone becomes more difficult.

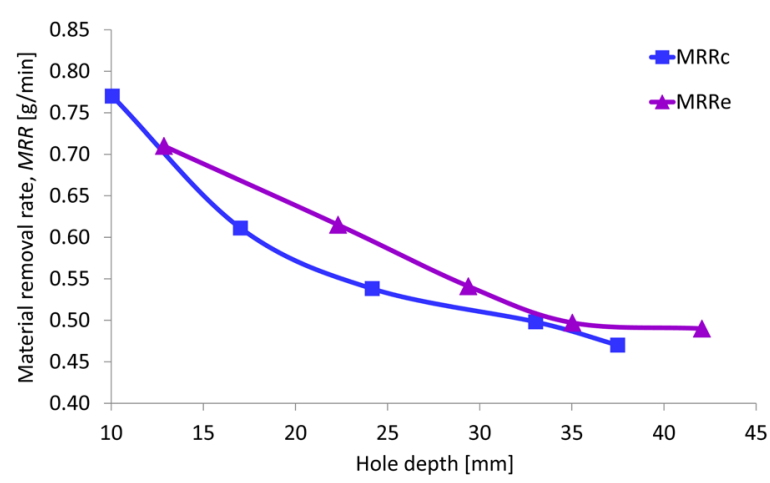

Fig. 5. MRR with respect to hole depth

$E W R$ is also an important issue related with $M R R$. Fig. 6 shows the EWR results for both electrodes. The elliptical electrode generally exhibits higher $E W R$, which is expected as the degree of erosion on both the workpiece and electrode are proportional to each other. Thus, providing high $M R R$, the elliptical electrode causes high EWR. However, the $E W R$ for the elliptical electrode decreases with an increase in hole depth while the EWR for the circular electrode increases due to weak flushing. This causes an accumulation of debris at the machining zone, leading to an increase in the degree of erosion of the electrode. The elliptical electrode has the advantage of providing its lowest degree of $E W R$, even in the case of deep holes.

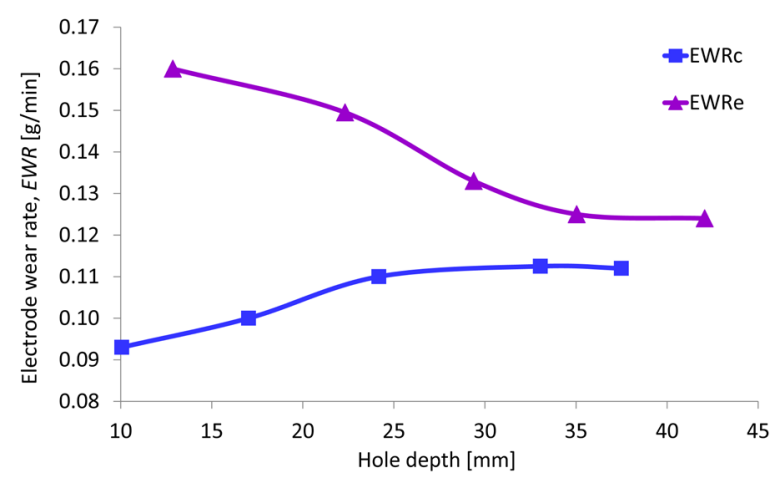

Fig. 6. EWR with respect to hole depth

$T W R$ is employed to evaluate the combined effect of $M R R$ and $E W R$. It is desirable to have lower $T W R$ values since this indicates a lesser amount of electrode wear and greater material removal. As seen in Fig. 7, a moderate increase in TWR is observed for the elliptical electrode whereas there is significant increase in the case of the circular electrode.

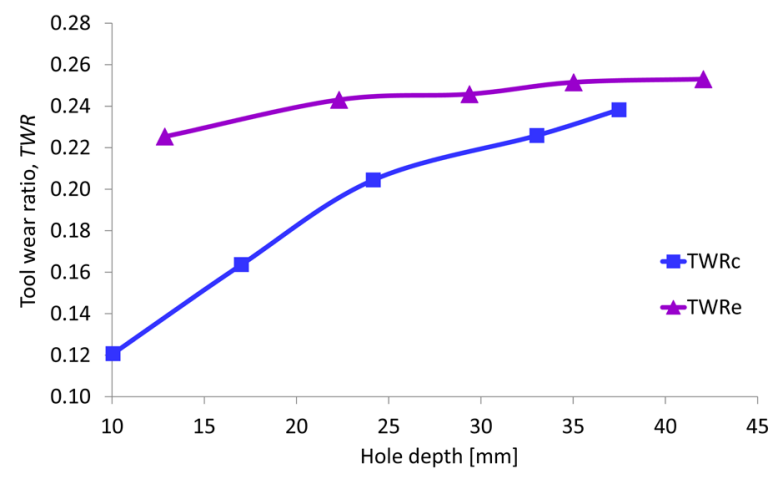

Fig. 7. TWR with respect to hole depth

\subsection{Dimensional Accuracy}

$A R$ is mainly related to the erosion capabilities of electrodes. $M R R$ for each electrode geometry was different, and hence different drilling depths were obtained for each hole. The results in Fig. 8 reveal that the elliptical electrode provided holes with higher $A R$, corresponding to greater amounts of material removal within specified drilling times, and hence deeper holes were produced.

Together with $A R, O C$ is also an important issue with regards to dimensional accuracy. Fig. 9 presents the results of $O C$. It is desirable that $O C$ should be the minimum for producing accurate holes. As seen in Fig. 9, the elliptical electrode exhibits lower $O C$ for all holes. In the case of the circular electrode, sparks are generated along the perimeter of electrode-workpiece gap. This leads to the removal of material on the side- 
walls of the drilled hole surface, causing enlargement in the hole diameter. Moreover, the amount of heat occurring in the machining zone in the case of the elliptical electrode is less than that of the circular electrode due to the improved flow of dielectric fluid through the larger gaps, decreasing the production of side-sparking and leading to lower $O C$.

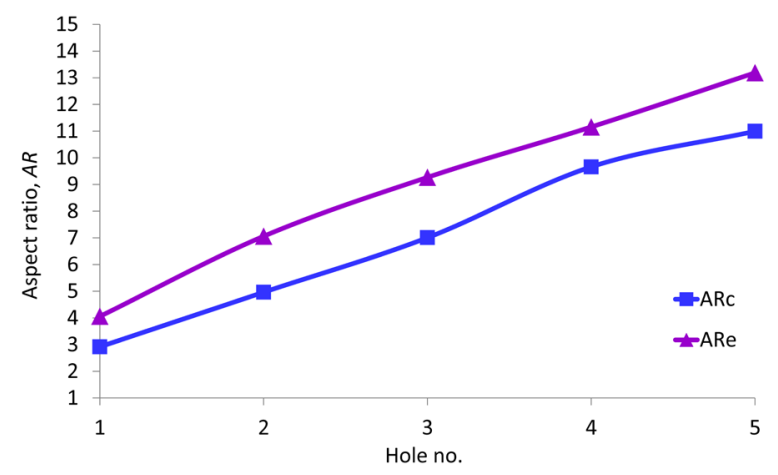

Fig. 8. AR with respect to hole number

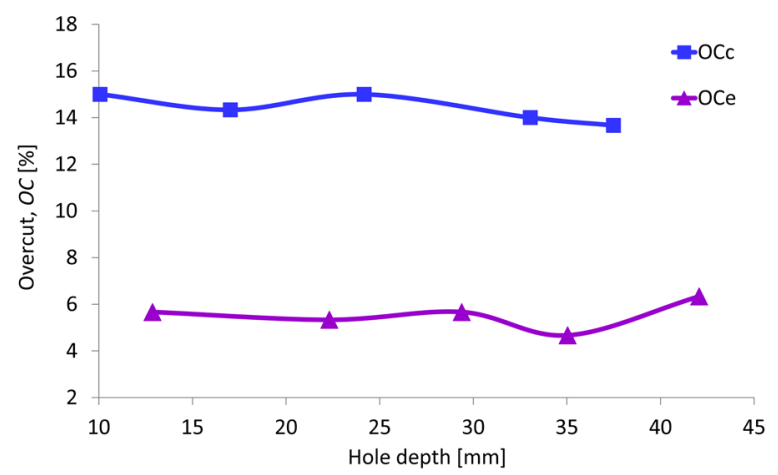

Fig. 9. OC with respect to hole depth
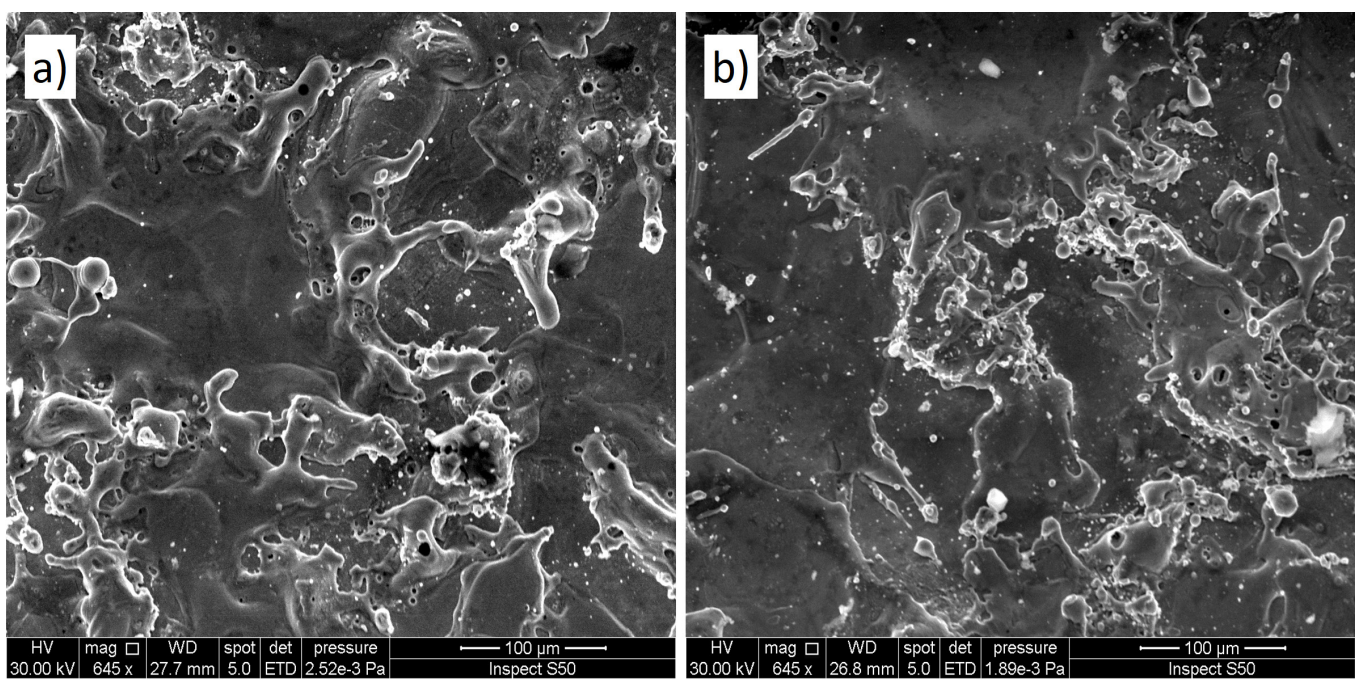

Fig. 11. SEM photographs of drilled surfaces; a) circular, and b) elliptical

\subsection{Surface Quality}

The comparison for $S R$ results is given in Fig. 10 . Regardless of the hole depth, the elliptical electrode provides smoother hole surfaces. This is owing to the good flushing capabilities of the elliptical electrode so that eroded particles can be effectively washed away without sticking to the workpiece surface, resulting in surfaces free from re-solidified debris.

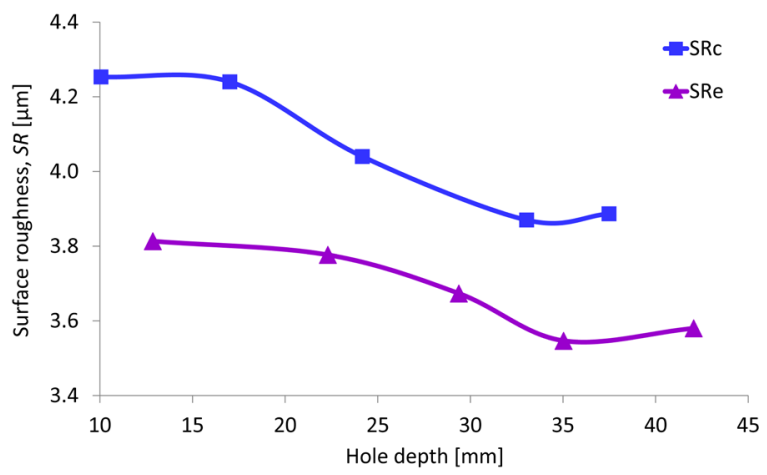

Fig. 10. SR with respect to hole depth

This conclusion is confirmed by means of the surface topography of drilled hole surfaces (Fig. 11). In discharge drilling, sparking causes significant melting and vaporization of particles (i.e. debris). This debris solidifies when it comes in contact with dielectric fluid. Due to insufficient flushing, debris cannot be washed away, and it sticks to the workpiece surface. This leads to the formation of surface anomalies (white layers and pockmarks), which adversely affect the surface quality. It is observed from Fig. 11 that the 
surface drilled with the elliptical electrode provided a lesser amount of white layer formation. This is due to improved flushing in case of elliptical electrode, and hence eroded particles were easily flushed away.

\section{NUMERICAL ANALYSES}

\subsection{Theoretical Background}

Flow characteristics were analysed based on a threephase model of flushing (i.e. water as liquid phase, bubbles as gas phase, and debris as solid phase) in ANSYS FLUENT. The interaction between bubbles and water was simulated with a Euler-Euler VOF model [16] to [18] by solving the volume continuity equation as follows [19]:

$$
\frac{\partial y}{\partial t}\left(\alpha_{g} \rho_{g}\right)+\nabla \cdot\left(\alpha_{g} \rho_{g} v_{g}\right)=\sum_{L=1}^{n}\left(m_{L g}\right)
$$

where $\alpha_{g}$ is the volume fraction of gas, $\rho_{g}$ is the density of gas, $v_{\mathrm{g}}$ is the velocity of gas, $m_{L g}$ is the mass transfer from the liquid phase to gas phase. The function of DEFIEN MASS TRANSFER was used to describe the transfer of water into gas [17]. When discharge occurs, water starts being transferred into bubbles. Processing of the water volume fraction $\left(\alpha_{w}\right)$ was computed with the following equation [19]:

$$
\alpha_{g}+\alpha_{L}=1
$$

For the VOF model, a single momentum conservation equation of the entire flow field was solved. The momentum equation was depending upon volume fractions of all phases through $\rho$ and $\mu$ [17]:

$$
\begin{gathered}
\frac{\partial}{\partial t}\left(\rho_{a} \mathrm{v}\right)+\nabla \cdot\left(\rho_{a} \mathrm{vv}\right)= \\
-\nabla p+\nabla \cdot\left[\mu_{a}\left(\nabla \mathrm{v}+\nabla \mathbf{v}^{T}\right)\right]+\rho_{a} g+F,
\end{gathered}
$$

where $\rho_{a}$ and $\mu_{a}$ were taken as the average of volume fraction values of phases, and $F$ denotes external body forces (i.e. forces from interaction with the dispersed phases). The calculations of $\rho_{a}$ and $\mu_{a}$ for two phases are as follows:

$$
\begin{aligned}
& \rho_{a}=\alpha_{L} \rho_{L}+\alpha_{g} \rho_{g}, \\
& \mu_{a}=\alpha_{L} \mu_{L}+\alpha_{g} \mu_{g},
\end{aligned}
$$

where $\alpha_{L}$ and $\alpha_{g}$ are the volume fractions of the liquid and gas phases, respectively.

Debris moves with the dielectric fluid in the machining gap, and hence its movement is abided by a discrete phase particle by integrating the force balance on the debris, which is written in a Lagrangian reference frame. The motion equation of the debris in the gap flow can be determined by Newton's second law of motion [17]:

$$
\frac{d u_{p}}{d t}=\frac{g_{z}\left(\rho_{p}-\rho_{L}\right)}{\rho_{p}}+F_{z}+F_{D}\left(u_{L}-u_{p}\right),
$$

where $u_{L}$ and $u_{p}$ are the velocities of water and debris in the direction of electrode movement, $\rho_{L}$ and $\rho_{p}$ are the densities of water and debris, $g_{z}$ is the gravitational acceleration. The first term on the right side of Eq. (11) is the buoyancy of debris. The second term is an additional force (thermo-phoresis force and Brownian force), including forces on particles arising due to the rotation of reference frame. The third term is the drag force of the debris, in which $F_{D}$ was calculated as follows [17]:

$$
F_{D}=\frac{18 \mu}{\rho_{p} d_{p}^{2}} \frac{C_{D} R_{e}}{24},
$$

where $\mu$ is the molecular viscosity of fluid, $d_{p}$ is the diameter of debris particles, $R_{e}$ is Reynolds number, $C_{D}$ is the coefficient of drag force.

The energy equation was also solved for the VOF model as below [22]:

$$
\frac{\partial}{\partial t}(\rho E)+\nabla \cdot(v(\rho E+p))=\nabla \cdot\left(k_{e f f} \nabla T\right)+S_{h} .
$$

The VOF model treats energy $(E)$ and temperature $(T)$ as mass-averaged variables:

$$
E=\frac{\sum_{q=1}^{n} \alpha_{q} \rho_{q} E_{q}}{\sum_{q=1}^{n} \alpha_{q} \rho_{q}},
$$

where $E_{q}$ for each phase is based on the specific heat of that phase and the shared temperature. The properties of $\rho$ and $k_{\text {eff }}$ (effective thermal conductivity) are shared by phases. The source term $\left(S_{h}\right)$ contains contributions from radiation as well as any other volumetric heat sources.

\subsection{Construction of Models}

3D models of the flow field during consecutive-pulse discharge was created. The deionized water (i.e. liquid phase) was introduced through the inner portion of the hollow electrode. The air (i.e. bubbles occurring due to vaporization) and the debris (i.e. particles eroded from workpiece) were generated within the gap between the electrode tip and the workpiece. The model equations given in the previous section were solved using a commercial CFD software package, FLUENT (v16.1). 
CFD simulations were carried out by means of GAMBIT tools in order to design the problem in geometrical configuration with an appropriate mesh. Before solving fluid flow problems, FLUENT needs the domain at which the flow takes place. Thereby, 3D flow domains for both electrode geometries were created in Solidworks ${ }^{\circledR}$. After that, the geometric model of the flow field domain inside the electrode and within the machining gap was divided into a certain number of cells (intervals) using GAMBIT software, and then imported into FLUENT. Solid models and the corresponding meshing for both electrode geometries are shown in Figs. 12 and 13, respectively.
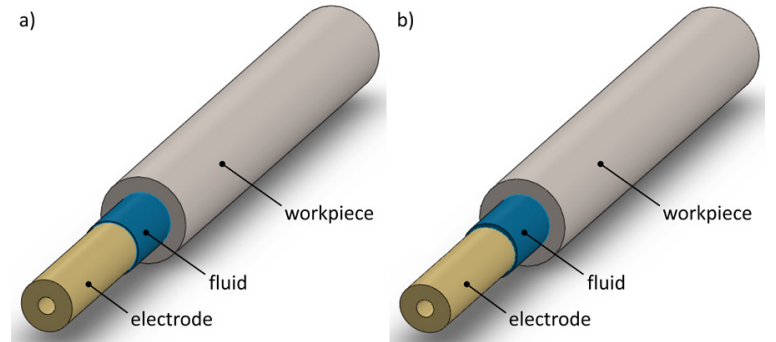

Fig. 12. Solid models representing electrode-fluid-workpiece interaction; a) circular, and b) elliptical
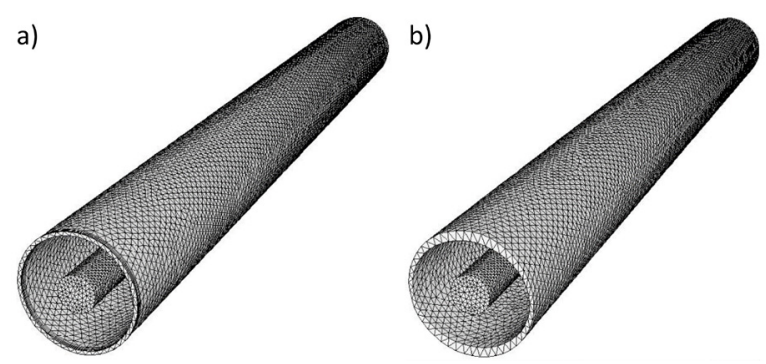

Fig. 13. Meshed models representing geometry at flushing gap; a) circular, and b) elliptical

To obtain well-posed system of equations, reasonable boundary conditions for the computational domain were implemented. The gap between the electrode and workpiece was initially filled with liquid (i.e. deionized water). Air bubbles and debris started to occur at the bottom region of the electrode, resulting from the sparks generated at the tip of the electrode, which melts the metal and boils the water. The outlet boundary condition was pressure that was set as $1.013 \times 10^{5} \mathrm{~Pa}$ (i.e. atmospheric pressure). The pressure boundary condition was applied at the top of electrode with a static reference pressure of $50 \mathrm{~kg} / \mathrm{cm}^{2}$. The inlet velocity was calculated as:

$$
\dot{m}=u_{i} A,
$$

where is the mass flow rate of water escaping from machining zone per unit time, and $A$ is the inside area of the hollow electrode. The inlet velocity $\left(u_{i}\right)$ was about $2 \mathrm{~m} / \mathrm{s}$. Wall boundary conditions were defined as a no-slip condition for the liquid but free-slip conditions for the solid and gas phases. The side and bottom faces of electrodes were rotated at $200 \mathrm{rpm}$. Identical boundary conditions were applied for both electrode geometries.

Simulations were carried out using FLUENT software based on the setup illustrated in Fig. 14. The maximum machining depth was $50 \mathrm{~mm}$. Side and bottom gaps were measured as suggested in the literature [18]. The difference between diameters of the electrode and drilled hole was the dimension of the side gap; it was found to be about $100 \mu \mathrm{m}$. The dimension of bottom gap was determined based on the difference between the vertical coordinates of the electrode. The coordinate of the electrode tip was recorded when a spark occurred between the electrode and workpiece. Without sparking, its coordinate was also recorded when the electrode was in contact with the workpiece. The difference between these two coordinates gives the dimension of the bottom gap, which was found to be $480 \mu \mathrm{m}$.

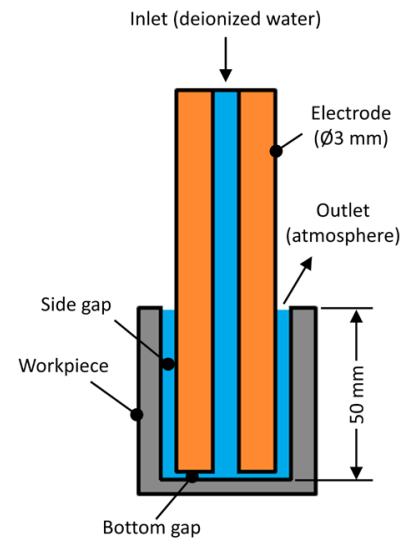

Fig. 14. Sketch of setup for numerical model

To verify the flow type of flow field in the machining gap (i.e. laminar or turbulent flow), the Reynolds number $\left(R_{e}\right)$ was calculated:

$$
R_{e}=\frac{u L}{v}
$$

where $u$ is the average speed of fluid flowing through the cross section of the machining gap, $L$ is the characteristic length of the flow field, $v$ is the kinematic viscosity of the water at the machining gap.

For the side gap; the characteristic length was constant $(0.1 \mathrm{~mm}), v$ was considered as kinematic 
viscosity of water (i.e. $0.801 \times 10^{-6} \mathrm{~m}^{2} / \mathrm{s}$ ), $v$ was considered as the speed of water at the side gap as calculated above. Thus, the maximum Reynolds number of the side gap was calculated as 413. This value is much smaller than the critical value of 2300; therefore, flow was said to be laminar.

Table 3. Properties for phases

\begin{tabular}{lccc}
\hline Property & $\begin{array}{c}\text { Liquid } \\
\text { (water) }\end{array}$ & $\begin{array}{c}\text { Solid } \\
\text { (AlSI 304) }\end{array}$ & $\begin{array}{c}\text { Gas } \\
\text { (air) }\end{array}$ \\
\hline Density $\left[\mathrm{kg} / \mathrm{m}^{3}\right]$ & 998.2 & 7000 & 1.225 \\
\hline Conductivity $[\mathrm{W} /(\mathrm{m} \cdot \mathrm{K})]$ & 0.6 & 17 & 0.0242 \\
\hline Specific Heat $[\mathrm{J} /(\mathrm{kg} \cdot \mathrm{K})]$ & 4182 & 530 & 1006.43 \\
\hline Viscosity $[\mathrm{kg} /(\mathrm{m} \cdot \mathrm{s})]$ & $100.3 \mathrm{E}-05$ & - & $1.7894 \mathrm{E}-05$ \\
\hline
\end{tabular}

In the discrete phase model, debris was assumed to have a spherical shape with diameter of $25 \mu \mathrm{m}$ [17]. The phase properties are given in Table 3 .

\subsection{Results and Discussions}

Numerical simulations of the inter-electrode gap were carried out to show the effectiveness of electrode shape on the behaviour of air bubbles and debris distribution at incremental time steps. Firstly, bubble distributions were simulated to define the motion of air bubbles from the bottom gap to the side gap. Fig. 15 shows the simulation results of the volume fraction at increasing time steps of $0.001 \mathrm{~s}, 0.005 \mathrm{~s}$, and 0.01 s. The left column of numerals with different colour fringes correspond to the volume fraction of bubbles. For instance, the blue regions denoted by " 0 " are full of water. The results in Fig. 15 reveal that the circular electrode exhibits uniformly distributed areas of bubbles around the side gap. In contrast, the bubble distribution is nonuniform in the case of the elliptical electrode, owing to its elliptical shape. The regions within the sides of larger gap, due to the elliptical shape, provide extra space for bubbles to flow, leading to stimulated motion of bubbles, which results in effective flushing capabilities.

Similar effects were observed for the debris distributions, as presented in Fig. 16. The left column of numerals with different colour fringes correspond to the accumulated moving time of debris. Regardless of the directions of motion of the debris and velocity fields, the dispersion degree of debris in the case of the circular electrode is higher than that of the elliptical electrode. It can be seen that the flushing of debris was increased when the elliptical electrode was used. Simulation results reveal that the elliptical electrode has a significant influence on the debris exclusion from the machining zone, and thus it has exhibited better flushing characteristics than the circular electrode has.

Debris concentrations along the hole depth are shown in Fig. 17. It is revealed that the elliptical electrode exhibits a higher level of debris exclusion towards the outlet section of hole, which is far from the bottom of hole (i.e. at the depth of $20 \mathrm{~mm}$ ). In contrast, in the case of the circular electrode, debris exclusion is diminished before $15 \mathrm{~mm}$. This proves that the elliptical electrode has good flushing capabilities for effective debris removal, even at deep hole regions.
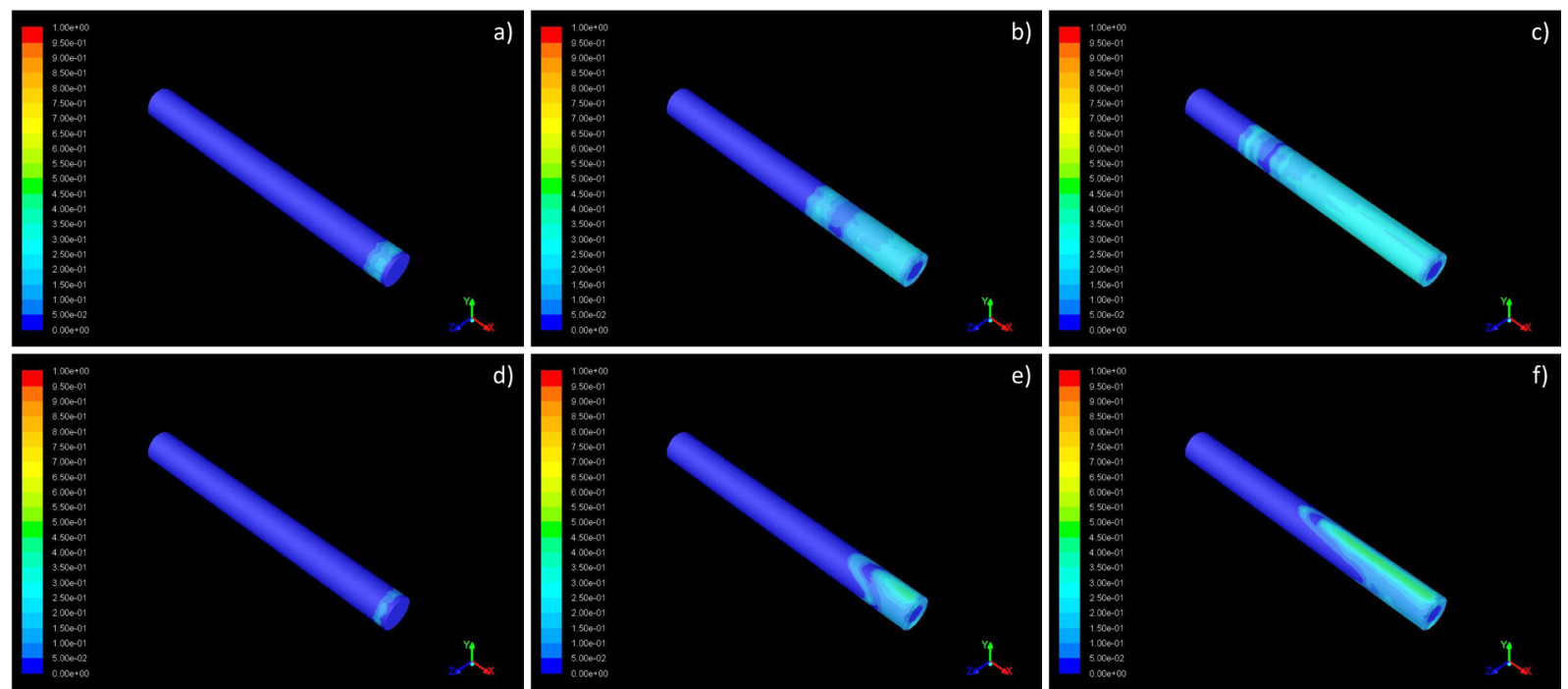

Fig. 15. Volume fraction contours of bubbles; a) $t=0.001 \mathrm{~s}, b) t=0.005 \mathrm{~s}, \mathrm{c}) t=0.01 \mathrm{~s}$ at circular electrodes, and d) $t=0.001 \mathrm{~s}$, e) $t=0.005 \mathrm{~s}, \mathrm{f}) t=0.01 \mathrm{~s}$ at elliptical electrodes 

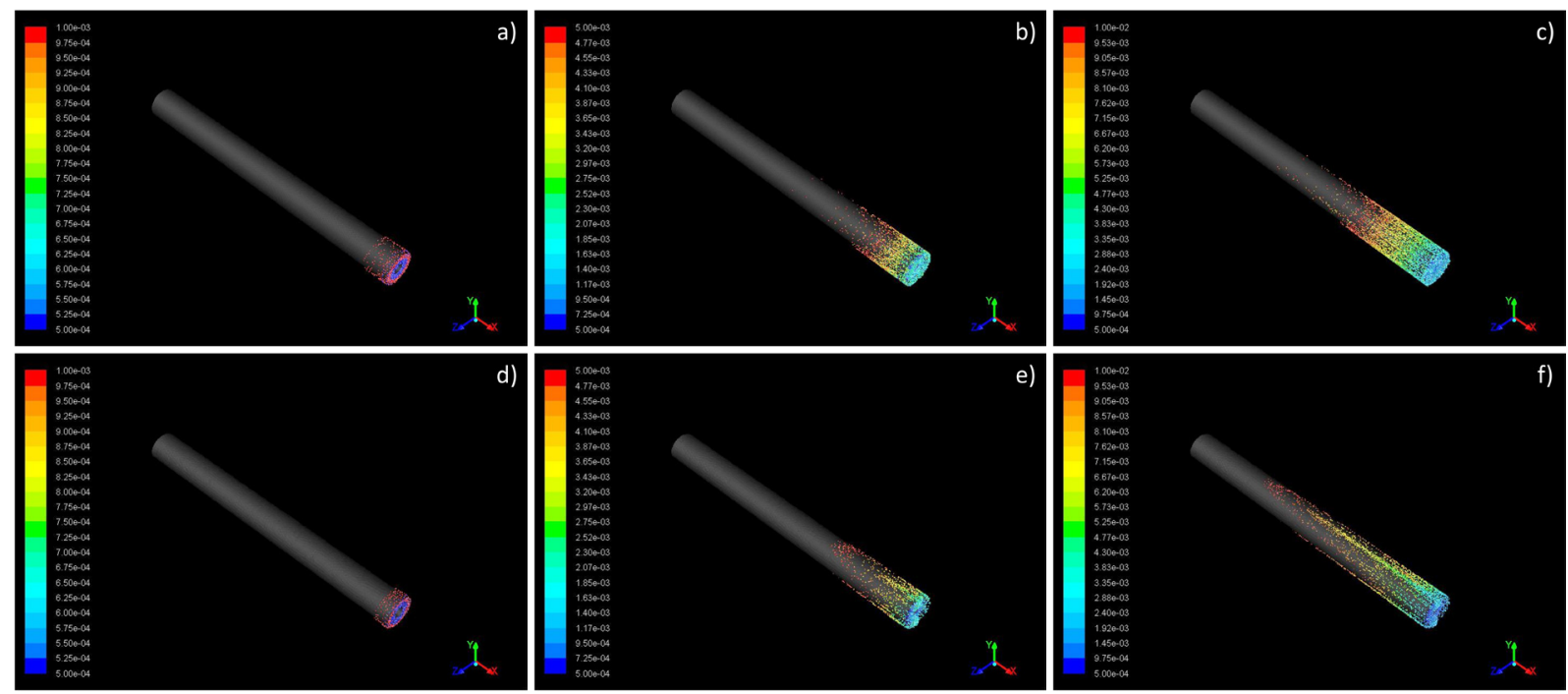

Fig. 16. Debris distributions; a) $t=0.001 \mathrm{~s}, b) t=0.005 \mathrm{~s}, \mathrm{c}) \mathrm{t}=0.01 \mathrm{~s}$ at circular electrodes, and d) $t=0.001 \mathrm{~s}, \mathrm{e}) t=0.005 \mathrm{~s}, \mathrm{f}) t=0.01 \mathrm{~s}$ at elliptical electrodes
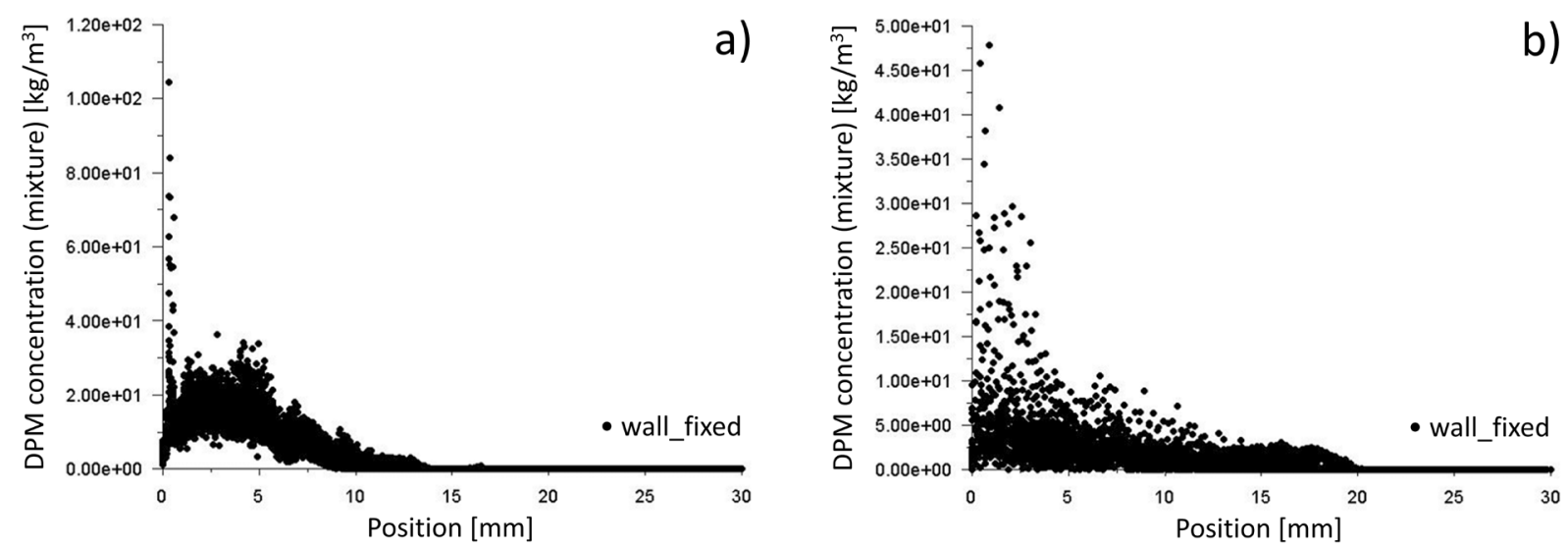

Fig. 17. Discrete phase models for debris concentration along the hole depth; a) circular, and b) elliptical

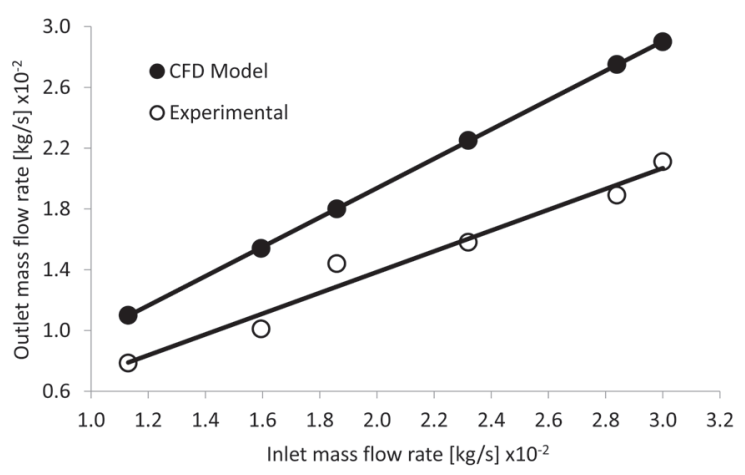

Fig. 18. Validation of MFR

The reliability of the developed models was validated based on the mass flow rate (MFR). For this purpose, several tests were conducted at varying drilling depths (at a range of $1 \mathrm{~mm}$ to $50 \mathrm{~mm}$ ) and inlet MFR values were measured. The corresponding outlet MFR values from the models were compared with experimental values. Fig. 18 shows that there is good agreement between the experimental and numerical MFR values. The CFD model has provided a linear correlation with high coefficient of determination (i.e. $R^{2}=0.969$ ). This assures the reliability of the developed model for the accurate simulation of the flow field at the interelectrode gap.

\section{CONCLUSIONS}

In this research, circular and elliptical hollow electrodes were used in drilling of Ø $3 \mathrm{~mm}$ holes on AISI 304 using an EDD process. Several drilling operations were conducted at varying durations. 
Numerical models were developed for the analysis of flushing capabilities. Both the experimental and numerical results revealed that electrode shape has significant effects on machining performance as well as the dimensional and surface characteristics of the drilled holes.

The main outcomes of this study are as follows:

- Regarding machining performance, the elliptical electrode provided greater MRR and EWR in comparison to the circular electrode. Similar results were observed for TWR. The modifications of electrode shape affected the erosion characteristics of the workpiece and electrode, leading to changes in material removal and electrode wear.

- In the assessment of dimensional features, holes with higher aspect ratios (i.e. deeper holes) were obtained with the elliptical electrode. Owing to its elliptical shape, spark intensity was increased at the bottom interelectrode gap, causing greater amounts of material removal (i.e. deeper holes). The elliptical electrode also provided holes with lower OC. This is because of the narrower contour along the perimeter of elliptical electrode, leading to reduced side-sparking, resulting in smaller enlargement of hole diameters.

- Regarding the surface quality of the drilled holes, the elliptical electrode provided lower SR values. It has better flushing performance due to the extra space at the side interelectrode gap, allowing for the easier removal of debris so that smoother hole surfaces were obtained.

- The numerical results based on the 3D three-phase models also proved that the elliptical electrode has good flushing capabilities for the efficient removal of air bubbles and eroded particles.

Despite high electrode wear, the overall performance of the elliptical electrode over the circular electrode was superior due to the improved erosion and flushing capabilities. Elliptical electrode provided holes with higher aspect ratio (up to 42 $\%$ ), less overcut (down to $66 \%$ ), and lower surface roughness (down to $7 \%$ ). The elliptical electrode is preferable for producing deep holes with good dimensional accuracy and high surface quality.

\section{REFERENCES}

[1] Sarikaya, M., Yilmaz, V. (2016). Optimization and predictive modeling using S/N, RSM, RA and ANNs for micro-electrical discharge drilling of AISI 304 stainless steel. Neural Computing and Applications, p. 1-15, D0l:10.1007/s00521016-2775-9.
[2] Bozdana, A.T., Ulutas, T. (2016). The effectiveness of multichannel electrodes on drilling blind holes on Inconel 718 by EDM process. Materials and Manufacturing Processes, vol. 31, no. 4, p. 504-513, DOl:10.1080/10426914.2015.1059451.

[3] Yu, Z.Y., Zhang, Y., Li, J., Luan, J., Zhao, F., Guo, D. (2009). High aspect ratio micro-hole drilling aided with ultrasonic vibration and planetary movement of electrode by microEDM. CIRP Annals, vol. 58, no. 1, p. 213-216, D0l:10.1016/j. cirp.2009.03.111.

[4] Bamberg, E., Heamawatanachai, S. (2009). Orbital electrode actuation to improve efficiency of drilling micro-holes by microEDM. Journal of Materials Processing Technology, vol. 209, no. 4, p. 1826-1834, D0l:10.1016/J.jmatprotec.2008.04.044.

[5] Jahan, M.P., Wong, Y.S., Rahman, M. (2012). Evaluation of the effectiveness of low frequency workpiece vibration in deep-hole micro-EDM drilling of tungsten carbide. Journal of Manufacturing Processes, vol. 14, no. 3, p. 343-359, D0l:10.1016/j.jmapro.2012.07.001.

[6] Shabgard, M.R., Alenabi, H. (2015). Ultrasonic assisted electrical discharge machining of Ti-6Al-4V alloy. Materials and Manufacturing Processes, vol. 30, no. 8, p. 991-1000, DOI:10.1080/10426914.2015.1004686.

[7] Yahagi, Y., Koyano, T., Kunieda, M., Yang, X. (2012). Micro drilling EDM with high rotation speed of tool electrode using the electrostatic induction feeding method. Procedia CIRP, vol. 1, p. 162-165, D0I:10.1016/J.procir.2012.04.028.

[8] Yadav, U.S., Yadava, V. (2015). Experimental investigation on electrical discharge drilling of Ti-6Al-4V alloy. Machining Science and Technology, vol. 19, no. 4, p. 515-535, DOI:10.1080/10910344.2015.1085316.

[9] Dwivedi, A.P., Choudhury, S.K. (2016). Effect of tool rotation on MRR, TWR, and surface integrity of AISI-D3 steel using the rotary EDM process. Materials and Manufacturing Processes, vol. 31, no. 14, p. 1844-1852, Dol:10.1080/10426914.2016. 1140198.

[10] Munz, M., Risto, M., Haas, R. (2013). Specifics of flushing in electrical discharge drilling. Procedia CIRP, vol. 6, p. 83-88, D0l:10.1016/j.procir.2013.03.024.

[11] Selvarajan, L., Narayanan, C.S., Jeyapaul, R. (2015). Optimization of process parameters to improve form and orientation tolerances in EDM of MoSi2-SiC composites. Materials and Manufacturing Processes, vol. 30, no. 8, p. 954-960, DOI:10.1080/10426914.2014.962041.

[12] Selvarajan, L., Narayanan, C.S., JeyaPaul, R. (2016). Optimization of EDM parameters on machining Si3N4TiN composite for improving circularity, cylindricity, and perpendicularity. Materials and Manufacturing Processes, vol. 31, no. 4, p. 405-412, DOI:10.1080/10426914.2015.1058947.

[13] Nastasi, R., Koshy, P. (2014). Analysis and performance of slotted tools in electrical discharge drilling. CIRP Annals, vol. 63, no. 1, p. 205-208, Dol:10.1016/j.cirp.2014.03.054.

[14] Plaza, S., Sanchez, J.A., Perez, E., Gil, R., Izquierdo, B., Ortega, N., Pombo, I. (2014). Experimental study on micro EDM-drilling of Ti6Al4V using helical electrode. Precision Engineering, vol. 38, no. 4, p. 821-827, Dol:10.1016/j. precisioneng.2014.04.010.

[15] Hung, J.C., Lin, J.K., Yan, B.H., Liu, H.S., Ho, P.H. (2006). Using a helical micro-tool in micro-EDM combined with ultrasonic 
vibration for micro-hole machining. Journal of Micromechanics and Microengineering, vol. 16, no. 12, p. 2705-2713, DOl:10.1088/0960-1317/16/12/025.

[16] Xie, B., Zhang, Y., Zhang, J., Rend, S. (2015). Numerical study of debris distribution in ultrasonic assisted EDM of hole array under different amplitude and frequency. International Journal of Hybrid Information Technology, vol. 8, no. 5, p. 151-158, DOl:10.14257/ijhit.2015.8.5.17.

[17] Wang, J., Han, F. (2014). Simulation model of debris and bubble movement in consecutive-pulse discharge of electrical discharge machining. International Journal of Machine Tools and Manufacture, vol. 77, p. 56-65, D0l:10.1016/j. ijmachtools.2013.10.007.

[18] Wang, J., Han, F. (2014). Simulation model of debris and bubble movement in electrode jump of electrical discharge machining. International Journal of Advanced Manufacturing Technology, vol. 74, no. 5-8, p. 591-598, Dol:10.1007/s00170014-6008-z.
[19] Kong, W., Guo, C., Zhu, X. (2015). Simulation analysis of bubble motion under ultrasonic assisted electrical discharge machining. $3^{\text {rd }}$ International Conference on Machinery, Materials and Information Technology Applications, Atlantis Press, D0l:10.2991/icmmita-15.2015.320.

[20] Xie, B., Zhang, Y., Zhang, J., Dai, Y., Liu, X. (2015). Flow field simulation and experimental investigation of ultrasonic vibration assisted EDM holes array. International Journal of Control and Automation, vol. 8, no. 12, p. 419-424, DOl:10.14257/ijca.2015.8.12.38.

[21] Zhang, W., Liu, Y., Zhang, S., Ma, F., Wang, P., Yan, C. (2015). Research on the gap flow simulation of debris removal process for small hole EDM machining with Ti alloy. $4^{\text {th }}$ International Conference on Mechatronics, Materials, Chemistry and Computer Engineering, Atlantis Press, D0l:10.2991/ icmmcce-15.2015.409.

[22] Ansys FLUENT 12.0 (Theory Guide). Ansys Inc. (2009). 